\title{
Cauliflower Growth, Yield and Quality Response to Nitrogen Fertilization and Micronutrients Foliar Application in Newly Reclaimed Areas Ali, M. A. M. ${ }^{1}$; E. A. A. Yousef ${ }^{2}$ and I. N. Nasef ${ }^{2}$ \\ ${ }^{1}$ Department of Horticulture, Faculty of Agriculture, New Valley University, Egypt. \\ ${ }^{2}$ Department of Horticulture, Faculty of Agriculture, Suez Canal University, Egypt. Correspondence: tohamyy@yahoo.com
}

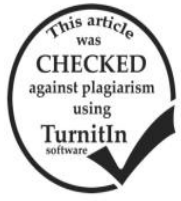

\section{ABSTRACT}

Two field experiments were carried out during 2017/2018 and 2018/2019 seasons, at the Experimental Farm, Faculty of Agriculture, New Valley University, New Valley governorate, Egypt, to study the effect of nitrogen levels (30, 45 and $60 \mathrm{~kg} / \mathrm{fed}$.) and micronutrients foliar application: control (B $0+\mathrm{Mo} 0)$, boron at $100 \mathrm{ppm}$ (B 100), molybdenum at $50 \mathrm{ppm}$ (Mo 50) and combined boron and molybdenum (B $100+$ Mo 50) on cauliflower vegetative growth, yield, and quality parameters in the recently reclaimed areas, especially New Valley governorate as a promising area for agriculture expansion in Egypt. The results revealed that nearly all traits showed a gradual increment with increasing the nitrogen level; whereas the application of high level of nitrogen (60 kg/fed.) significantly increased vegetative growth, curd yield and curd quality of cauliflower and significantly decreased total phenols content and has no effect on number of days to maturity. Also, the results of this study reported promotional effects of either B or Mo foliar application on growth, yield and quality of cauliflower curds compared with untreated plants; nevertheless the combined application of B and Mo together was more effective than application of B or Mo when they separately applied. Moreover, inorganic nitrogen at $60 \mathrm{~kg} / \mathrm{fed}$. was more effective in the presence of B and Mo at 100 and $50 \mathrm{ppm}$, respectively, than the other combinations and achieved the highest curd yield with top quality.

Keywords: Brassica oleraceae, nitrogen level, boron, molybdenum

\section{INTRODUCTION}

Cauliflower (Brassica oleracea L. var. botrytis) is an important cool season vegetable crop and belongs to Brassicaceae family. It is one of the highest antioxidative activity vegetables because of its high content of phytochemicals, such as glucosinolates, vitamins, phenolic compounds and fibers, which are principals for digestive system health (Podsedek, 2007; Picchi et al., 2012). The health promoting properties of cauliflower have been strongly emphasized and were referred to its high content of sulphoraphane, indole-3-carbinol and 2-propenyl isothiocyanates, which are the breakdown products of glucoraphanin, glucobrassicin and sinigrin, respectively (Agerbirk et al., 2009). Therefore, cauliflower has been shown to be very effective in protecting against the risk of several types of cancer (Lee et al., 2008; Tang et al., 2008).

Like other crops, plant nutrition is one of the key principals for getting high yield of cauliflower. It is well known to be a heavy feeder crop and has the capacity to absorb high amount of macronutrients, particularly nitrogen (Abdel-Razzak et al., 2008 a; Bianco et al., 2015). Nitrogen $(\mathrm{N})$ significantly affects the crop productivity as well as quality attributes (Konstantopoulou et al., 2010; He-xi et al., 2011), through controlling the synthesis of several key products in plant cell, such as nucleic acids, proteins, phospholipids and other secondary metabolites (Amtmann and Armengaud, 2009). The optimum supply of $\mathrm{N}$ enhances plant growth and crop productivity (Collins and McCoy, 1997); however, the excessive and overuse of $\mathrm{N}$ fertilization may increase the accumulation of compounds such as nitrates and non protein nitrogen in the edible parts, which may be harmful to human health and cause several environmental pollution and economical losses (Lisiewska and Kmiecik, 1996; Elia et al., 1998; Rani and Mallareddy, 2007; Elwan and El-Shatoury, 2012). Pervious investigations have showed that curd yield and quality of cauliflower and broccoli are greatly influenced by $\mathrm{N}$ application (Abdel-Razzak et al., 2008 a and b; Yoldas et al., 2008; Abd El-All and EL-Shabrawy,
2013; Singh et al., 2018). Therefore, supply of proper N must be ensured during cauliflower cultivation.

In addition, micronutrients are essential elements for normal plant growth, development and productivity of plants as they play important roles in the meristematic development, chlorophyll formation, photosynthesis and transpiration as well as tannin and phenolic compound development (Sharma, 2006; Tripathi et al., 2015). Boron (B) is very important for growth and development of crops as it is involved in cell division, root elongation, calcium metabolism, auxin synthesis, sugar metabolism translocation of solutes and protein synthesis (Tariq and Mott, 2007; Camacho-Cristóbal et al., 2018). Molybdenum (Mo) is also an important micronutrient for plant growth and development. It is an essential component of major enzyme, nitrate reductase, which converts the nitrate to nitrite, which is the first stage of the incorporation of nitrogen to proteins (Kaiser et al., 2005; Bambara and Ndakidemi, 2010).

Some vegetable crops have higher demand micronutrients than others and should be applied in optimum rates. Cauliflower has a high micronutrients requirement, particularly B and Mo. Several previous studies reported that both B and Mo are important elements in attributes of cauliflower and broccoli productivity and quality (Thapa et al., 2015; Hassain et al., 2018; Sarker et al., 2018). Unfortunately, B and Mo deficiency has been reported very frequently in cauliflower and caused several anatomical, physiological, and biological changes, such as browning of curd and hollow stem as well as whiptail disorders, sword like leaves, malformation of growing tip and delaying curd formation (Mehrotra and Mishra, 1974; Shelp and Liu, 1992; Sharma, 2002). In addition, the affected heads become irregular in shape, smaller in size, and bitter in taste, which adversely affects the market demand of the crop. Nevertheless, several researchers reported that B and Mo deficiency could be overcome by application of B and Mo, either as soil application or as foliar spray, and enhanced the cauliflower growth and productivity (Ahmed et al., 2011; Ningawale et al., 2016; Farooq et al., 2018). Therefore, application of B and Mo is a crucial factor for yield and quality of cauliflower. 
In last decades, the rapid growth of population is one of the main challenges facing Egypt. In order to overcome this problem, policy makers are trying to expand the arable land, which represents only $3.5 \%$ of total area, into the new rejoins such as recently reclaimed areas over all the country. However, information regarding the standard cultural practices such as fertilization requirements is still meager in these zones. Keeping this view in perspectives, the following field experiments were performed with a view to find out the optimum level of $\mathrm{N}$ and foliar application of $\mathrm{B}$ and Mo for obtaining maximum growth, yield and quality of cauliflower in newly reclaimed area in the south-west part of Egypt.

\section{MATERIALS AND METHODS}

\section{Plant materials and treatments}

Two field experiments were performed out in consecutive winter seasons of 2017 and 2018 at Experimental Research Farm of the Faculty of Agriculture, New Valley University, New Valley governorate, Egypt in order to study the effect of $\mathrm{N}$ levels and foliar application of micronutrients: $\mathrm{B}$ and Mo as well as their interaction on vegetative growth, yield, quality and chemical components of cauliflower "cv. Amshiry". N was supplied as ammonium sulphate at level of 30, 45 (according to recommendation of Agriculture Ministry of Egypt) and $60 \mathrm{~kg} \mathrm{~N} / \mathrm{fed}$. Four micronutrients treatments were supplied: (1) control (B 0 + Mo 0), (2) B at 100 ppm (B 100; borax was used as source for boron), (3) Mo at $50 \mathrm{ppm}$ (Mo 50; ammonium molybdate was used as a source for molybdenum) and (4) boron at $100 \mathrm{ppm}$ plus molybdenum at 50 ppm together (B 100 + Mo 50). Both treatments and their interactions were arranged in a split plot in randomized complete block design with three replications. $\mathrm{N}$ levels were applied at three doses; the first dose was applied after four weeks from transplantation and the rest doses were supplied with three weeks intervals. The treatments of micronutrients with three drops of tween-twenty were sprayed four times.

The first micronutrient treatment was applied at age 30 after transplantation with two weeks intervals on the whole foliage in the morning (10-11 am). The plants that were treated with combined application of B + Mo were firstly sprayed with B and in the next day were sprayed with Mo. Depending on plant development stage, the volume of sprayed solution ranged from $20 \mathrm{ml}$ to $50 \mathrm{ml}$ per plant each time with a manual pump. The same amount of double distilled water plus three drops of tween-twenty was sprayed to the control plants.

\section{Experimental design}

Each replicate consisted of 12 treatments representing all combinations among the three levels of nitrogen and four treatments of micronutrients. The main plots were assigned to levels of nitrogen levels, while micronutrients treatments occupied as sub-plot and were placed randomly in each main plot. The experimental unit area (plot) was $11.2 \mathrm{~m}^{2}$ in size and contained 4 ridges (with $4 \mathrm{~m}$ in length and $0.7 \mathrm{~m}$ in width for each ridge) and included 40 plants. A guard row was left without planting to separate each two adjacent sub-plots to protect against border effects.

\section{Soil analysis}

Before plantation, five soil samples were randomly collected at depth of $0.0-40.0 \mathrm{~cm}$ and then homogenized together in order to determine the physicochemical and chemical characteristics (Jackson, 1973; Chapman and Pratt, 1978; Klute, 1986). The soil physicochemical and chemical characteristics of the experimental site are presented in Table (1).

Table 1. Physicochemical and chemical characteristics of the experimental site in growing seasons.

\begin{tabular}{|c|c|c|}
\hline $\begin{array}{l}\text { Soil } \\
\text { characteristics }\end{array}$ & $\begin{array}{c}\text { Season } \\
2107 / 2018\end{array}$ & $\begin{array}{c}\text { Season } \\
2018 / 2019\end{array}$ \\
\hline Silt (\%) & 12.77 & 12.54 \\
\hline Clay (\%) & 7.85 & 7.80 \\
\hline Sand (\%) & 79.38 & 79.66 \\
\hline Texture & Sandy & Sandy \\
\hline $\mathrm{PH}$ & 8.15 & 8.20 \\
\hline E.C dsm & 0.98 & 0.95 \\
\hline Organic matter $(\%)$ & 0.51 & 0.54 \\
\hline $\mathrm{CaCO}_{3}(\%)$ & 4.97 & 5.05 \\
\hline Available N (mg/kg) & 48.80 & 51.24 \\
\hline Available P (mg/kg) & 5.16 & 4.95 \\
\hline Available K (mg/kg) & 133.70 & 142.36 \\
\hline $\mathrm{Ca}^{++}(\mathrm{meq} / 100 \mathrm{~g})$ & 0.98 & 0.95 \\
\hline $\mathrm{Mg}^{++}$(meq/100g & 0.66 & 0.40 \\
\hline $\mathrm{Na}^{+}(\mathrm{meq} / 100 \mathrm{~g})$ & 3.15 & 3.21 \\
\hline $\mathrm{CO}_{3}^{--}(\mathrm{meq} / 100 \mathrm{~g})$ & - & - \\
\hline $\mathrm{HCO}_{3}^{-}(\mathrm{meq} / 100 \mathrm{~g})$ & 1.13 & 1.10 \\
\hline $\mathrm{Cl}^{-}(\mathrm{meq} / 100 \mathrm{~g})$ & 2.97 & 2.85 \\
\hline $\mathrm{SO}_{4}^{--}(\mathrm{meq} / 100 \mathrm{~g})$ & 0.92 & 0.88 \\
\hline
\end{tabular}

\section{Agricultural practices}

Seeds of cauliflower cv. "Amshiry" were firstly sown in 209-cell styrophom trays in greenhouse to produce the transplants. Trays were filed with soil mixture (peatmoss and vermiculite mixes in 1:1). The germination conditions were $22-26{ }^{\circ} \mathrm{C}$ and $85-90 \%$ relative humidity. During the seedling growth in the greenhouse, the experimental sites were prepared by adding $20 \mathrm{~m}^{3}$ of cattle manure in addition to $200 \mathrm{~kg} / \mathrm{fed}$. of calcium super phosphate $\left(15.5 \% \quad \mathrm{P}_{2} \mathrm{O}_{5}\right)$. Later, the soil was cleared, ploughed, harrowed and divided into plots. After 30 days, healthy cauliflower seedlings with 3-4 true leaves were transplanted by hand on one side of the ridge at a distance of $40 \mathrm{~cm}$ on October $17^{\text {th }}$ and $12^{\text {nd }}$ in 2017 and 2018 seasons, respectively. All cultural practices (i.e. irrigation, weed and pest control) used for the cauliflower production were performed according to instructions of the Egyptian Ministry of Agriculture for cauliflower field production.

\section{Measurements}

At maturity stage, five plants were randomly selected from each experimental unit (plot) and data were recorded as follows:

\section{A. Vegetative growth parameters:}

- Plant height, number of leaves, leaf area $\left(\mathrm{cm}^{2}\right)$, leaves fresh weight $(\mathrm{g})$, leaves dry matter $(\%)$.

\section{B. Yield parameters:}

- Curd fresh weight $(\mathrm{g})$, curd diameter $(\mathrm{cm})$, curd dry matter (\%), total curd yield/fed. (ton), number of days to maturity

C. Curd chemical quality parameters:

- Ascorbic acid (mg/100 g FW) was measured according to the method described by A.O.A.C. (1975).

- Carbohydrates content (\%): It was determined according to Mazumdar and Majumder (2003). 
- Total phenols content (mg/100 g FW): It was determined according to Mazumdar and Majumder (2003).

- Soluble solids content (SSC \%) was measured by a digital refractometer (Atago N1, Japan).

- Nitrate content (ppm) was measured according to Cataldo et al. (1975).

- Sulphur content (ppm) was estimated according to Novozamsky and Van Eck (1977).

D. Total Chlorophyll content and mineral composition in leaves:

- Total chlorophyll content (mg/g FW) was measured spectrophotometrically according to Lichenthaler and Wellburn (1983).

- N (\%) was measured according to method of Baethgen and Ally (1989).

- P (\%) was determined as described by Jackson (1973).

- K (\%) was determined as described by Page (1982).

- B (ppm) was determined by method of Dible et al. (1954).

- Mo (ppm) was determined by methods of Johnson and Arkley (1954).

Statistical analysis

The collected data were subjected to statistical analysis using two-ways analysis of variance (ANOVA) procedures as implemented in Co-Stat Software computer program for statistics (2004). Means for nitrogen levels and micronutrients treatments as well as their interactions were

Table

Table 2. Main effects of nitrogen levels, micronutrients treatments and their interactions on vegetative growth parameters of cauliflower

\begin{tabular}{|c|c|c|c|c|c|c|c|c|}
\hline \multirow{2}{*}{ Treatments } & \multicolumn{4}{|c|}{$2017 / 2018$ season } & \multicolumn{4}{|c|}{ 2018/2019 season } \\
\hline & N1 & N2 & $\mathrm{N3}$ & Mean & N1 & $\mathbf{N 2}$ & $\mathbf{N 3}$ & Mean \\
\hline & \multicolumn{8}{|c|}{ Plant height $(\mathrm{cm})$} \\
\hline $\mathrm{B} 0+\mathrm{Mo} 0$ & $47.27 \mathrm{i}$ & $48.26 \mathrm{hi}$ & $48.64 \mathrm{hi}$ & $48.05 \mathrm{D}$ & $47.91 \mathrm{j}$ & $48.62 \mathrm{ij}$ & $50.20 \mathrm{hi}$ & $48.91 \mathrm{D}$ \\
\hline B 100 & $49.75 \mathrm{gh}$ & $51.01 \mathrm{fg}$ & $52.30 \mathrm{ef}$ & $51.02 \mathrm{C}$ & $50.64 \mathrm{hi}$ & $51.95 \mathrm{gh}$ & $53.03 \mathrm{fg}$ & $51.87 \mathrm{C}$ \\
\hline Mo 50 & $53.68 \mathrm{de}$ & $55.96 \mathrm{~cd}$ & $58.57 \mathrm{ab}$ & $56.07 \mathrm{~B}$ & 54.24 ef & $56.98 \mathrm{~cd}$ & $59.61 \mathrm{ab}$ & $56.94 \mathrm{~B}$ \\
\hline B $100+$ Mo 50 & $54.79 \mathrm{~d}$ & $57.34 \mathrm{bc}$ & $60.47 \mathrm{a}$ & $57.53 \mathrm{~A}$ & $55.27 \mathrm{de}$ & $58.67 \mathrm{bc}$ & $61.72 \mathrm{a}$ & $58.55 \mathrm{~A}$ \\
\hline Mean & $51.37 \mathrm{C}$ & $53.14 \mathrm{~B}$ & $54.99 \mathrm{~A}$ & & $52.01 \mathrm{C}$ & $54.05 \mathrm{~B}$ & $56.14 \mathrm{~A}$ & \\
\hline \multicolumn{9}{|c|}{ Number of leaves/plant } \\
\hline B $0+$ Mo 0 & $17.33 \mathrm{f}$ & $18.00 \mathrm{ef}$ & $18.33 \mathrm{ef}$ & $17.88 \mathrm{C}$ & $17.66 \mathrm{f}$ & $18.33 \mathrm{ef}$ & $19.33 \mathrm{de}$ & $18.44 \mathrm{C}$ \\
\hline B 100 & $18.33 \mathrm{ef}$ & $19.00 \mathrm{de}$ & $19.00 \mathrm{de}$ & $18.77 \mathrm{~B}$ & $19.33 \mathrm{de}$ & $19.66 \mathrm{~d}$ & $20.00 \mathrm{~d}$ & $19.66 \mathrm{~B}$ \\
\hline Mo 50 & $19.66 \mathrm{~cd}$ & $20.33 \mathrm{bc}$ & $21.33 \mathrm{ab}$ & $20.44 \mathrm{~A}$ & $20.00 \mathrm{~d}$ & $21.33 \mathrm{bc}$ & $22.33 \mathrm{ab}$ & $21.22 \mathrm{~A}$ \\
\hline B $100+$ Mo 50 & $20.00 \mathrm{~cd}$ & $20.33 \mathrm{bc}$ & $21.66 \mathrm{a}$ & $20.66 \mathrm{~A}$ & $20.33 \mathrm{~cd}$ & $21.33 \mathrm{bc}$ & $22.66 \mathrm{a}$ & $21.44 \mathrm{~A}$ \\
\hline Mean & $18.83 \mathrm{C}$ & $19.41 \mathrm{~B}$ & $20.08 \mathrm{~A}$ & & $19.33 \mathrm{C}$ & $20.16 \mathrm{~B}$ & $21.08 \mathrm{~A}$ & \\
\hline \multicolumn{9}{|c|}{ Leaf area/plant $\left(\mathrm{cm}^{2}\right)$} \\
\hline B $0+$ Mo 0 & $7012.60 \mathrm{j}$ & $8621.39 \mathrm{i}$ & $9023.65 \mathrm{hi}$ & 8219.21 D & $8022.20 \mathrm{~h}$ & $8815.25 \mathrm{gh}$ & $9132.99 \mathrm{gh}$ & 8656.81 D \\
\hline & $9935.34 \mathrm{gh}$ & $10910.25 \mathrm{fg}$ & 11478.13 ef & $10774.57 \mathrm{C}$ & $10138.15 \mathrm{fg}$ & $10963.01 \mathrm{ef}$ & $11504.70 \mathrm{ef}$ & $10868.62 \mathrm{C}$ \\
\hline Mo 50 & 2388.84 de & $13410.77 \mathrm{~cd}$ & $15514.11 \mathrm{ab}$ & $13771.24 \mathrm{~B}$ & $12509.73 \mathrm{de}$ & $13925.85 \mathrm{~cd}$ & $16060.72 \mathrm{ab}$ & $14165.43 \mathrm{~B}$ \\
\hline B $100+$ Mo 50 & $12687.17 \mathrm{de}$ & $14589.83 \mathrm{bc}$ & $16757.27 \mathrm{a}$ & $14678.09 \mathrm{~A}$ & $13363.07 \mathrm{~d}$ & $15312.67 \mathrm{bc}$ & $17695.58 \mathrm{a}$ & $15457.11 \mathrm{~A}$ \\
\hline Mean & $10505.99 \mathrm{C}$ & $11883.06 \mathrm{~B}$ & $13193.29 \mathrm{~A}$ & & $11008.29 \mathrm{C}$ & $12254.19 \mathrm{~B}$ & $13598.50 \mathrm{~A}$ & \\
\hline \multicolumn{9}{|c|}{ Fresh weight of leaves/plant (g) } \\
\hline $\mathrm{B} 0+\mathrm{Mo} 0$ & $1117.16 \mathrm{j}$ & $1141.00 \mathrm{ij}$ & $1222.86 \mathrm{ij}$ & $1160.34 \mathrm{D}$ & $1128.63 \mathrm{i}$ & $1199.64 \mathrm{hi}$ & $1332.62 \mathrm{gh}$ & $1220.30 \mathrm{D}$ \\
\hline B 100 & $1276.92 \mathrm{hi}$ & $1373.13 \mathrm{gh}$ & $1436.43 \mathrm{fg}$ & $1362.16 \mathrm{C}$ & $1357.83 \mathrm{~g}$ & $1457.73 \mathrm{fg}$ & $1522.28 \mathrm{f}$ & $1445.95 \mathrm{C}$ \\
\hline Mo 50 & $1527.00 \mathrm{ef}$ & $1694.65 \mathrm{~cd}$ & $1851.10 \mathrm{ab}$ & $1690.91 \mathrm{~B}$ & $1581.38 \mathrm{ef}$ & $1746.96 \mathrm{~cd}$ & $1899.25 \mathrm{ab}$ & $1742.53 \mathrm{~B}$ \\
\hline B $100+$ Mo 50 & $1593.77 \mathrm{de}$ & $1786.38 \mathrm{bc}$ & $1963.27 \mathrm{a}$ & $1781.14 \mathrm{~A}$ & $1666.03 \mathrm{de}$ & $1836.58 \mathrm{bc}$ & $1998.27 \mathrm{a}$ & $1833.62 \mathrm{~A}$ \\
\hline Mean & $1378.71 \mathrm{C}$ & $1498.79 \mathrm{~B}$ & $1618.42 \mathrm{~A}$ & & $1433.47 \mathrm{C}$ & $1560.23 \mathrm{~B}$ & $1688.10 \mathrm{~A}$ & \\
\hline \multicolumn{9}{|c|}{ Dry matter of leaves/plant $\%$} \\
\hline$\overline{\mathrm{B} 0+\mathrm{Mo} 0}$ & $8.46 \mathrm{~g}$ & $9.36 \mathrm{f}$ & $9.82 \mathrm{ef}$ & $9.21 \mathrm{D}$ & $9.29 \mathrm{f}$ & 9.78 ef & 10.42 ef & $9.83 \mathrm{D}$ \\
\hline B 100 & $9.94 \mathrm{ef}$ & $10.27 \mathrm{de}$ & $10.33 \mathrm{de}$ & $10.18 \mathrm{C}$ & $10.62 \mathrm{de}$ & $10.67 \mathrm{de}$ & $10.73 \mathrm{de}$ & $10.67 \mathrm{C}$ \\
\hline Mo 50 & $10.40 \mathrm{de}$ & $11.45 \mathrm{c}$ & $12.64 \mathrm{ab}$ & $11.50 \mathrm{~B}$ & $10.81 \mathrm{de}$ & $12.14 b c$ & $13.02 \mathrm{ab}$ & $11.99 \mathrm{~B}$ \\
\hline B $100+$ Mo 50 & $10.97 \mathrm{~cd}$ & $11.80 \mathrm{bc}$ & $13.28 \mathrm{a}$ & $12.02 \mathrm{~A}$ & $11.77 \mathrm{~cd}$ & $12.77 \mathrm{bc}$ & $13.98 \mathrm{a}$ & $12.84 \mathrm{~A}$ \\
\hline Mean & $9.94 \mathrm{C}$ & $10.72 \mathrm{~B}$ & $11.51 \mathrm{~A}$ & & $10.62 \mathrm{C}$ & $11.34 \mathrm{~B}$ & $12.04 \mathrm{~A}$ & \\
\hline
\end{tabular}

Values are the means of three replicates. Values followed by the same letters within a column for each genus are not significantly different at the $1 \%$ level of probability according to Duncan's multiple range test. $\mathrm{N} 1=30 \mathrm{~kg}$ nitrogen/fed., $\mathrm{N} 2=45 \mathrm{~kg}$ nitrogen/fed., $\mathrm{N3}=$ $60 \mathrm{~kg}$ nitrogen/fed., B $0+$ Mo $0=$ control, B 100= $100 \mathrm{ppm}$ of boron, Mo 50=50 ppm of molybdenum and B $100+$ Mo $50=100 \mathrm{ppm}$ of boron combined with $50 \mathrm{ppm}$ of molybdenum. 
Effect of nitrogen levels as well as B and Mo on yield and yield parameters

Data in Table (3) clearly show that all yield traits had gradual increment with increasing nitrogen level up to $60 \mathrm{~kg} / \mathrm{fed}$. in both growing seasons except number of days to maturity. With regard to the main effect of micronutrient treatments, Table (3) shows that all yield traits were statistically affected by the application of all micronutrient treatments except number of days to maturity. The significant highest values of curd fresh weight, curd diameter, curd dry matter and curd yield were observed in plants subjected to B and Mo together, followed by the plant that received Mo at $50 \mathrm{ppm}$ alone. Nevertheless, the difference between these two treatments was not significant for curd diameter and curd dry matter in both growing seasons. Regarding to the interaction effect between nitrogen levels and micronutrient treatments, the results showed that cauliflower plants fertilized with $60 \mathrm{~kg} \mathrm{~N} / \mathrm{fed}$. and foliar sprayed with combined application of B and Mo at 100 and $50 \mathrm{ppm}$, respectively, had the significant highest curd fresh weight, curd diameter, curd dry matter and curd yield in comparison with other combinations; however, this combination had no effect on the number of days to maturity. The above-mentioned treatment enhanced curd yield by $73.06 \%$ and $74.27 \%$ in season of 2017/2018 and 2018/2019, respectively.

Table 3. Main effects of nitrogen levels, micronutrients treatments and their interactions on yield and yield components of cauliflower.

\begin{tabular}{|c|c|c|c|c|c|c|c|c|}
\hline \multirow{2}{*}{ Treatments } & \multicolumn{4}{|c|}{ 2017/2018 season } & \multicolumn{4}{|c|}{ 2018/2019 season } \\
\hline & N1 & N2 & N3 & Mean & N1 & N2 & N3 & Mean \\
\hline \multicolumn{9}{|c|}{ Curd fresh weight (g) } \\
\hline B $0+$ Mo 0 & $1128.92 \mathrm{~h}$ & $1289.40 \mathrm{gh}$ & $1367.57 \mathrm{fg}$ & $1261.96 \mathrm{D}$ & $1246.92 \mathrm{~h}$ & $1293.02 \mathrm{gh}$ & $1368.33 \mathrm{gh}$ & $1302.75 \mathrm{D}$ \\
\hline B 100 & $1402.91 \mathrm{fg}$ & $1488.78 \mathrm{ef}$ & $1508.14 \mathrm{ef}$ & $1466.61 \mathrm{C}$ & $1425.39 \mathrm{fg}$ & $1541.51 \mathrm{f}$ & $1587.01 \mathrm{ef}$ & $1517.97 \mathrm{C}$ \\
\hline Mo 50 & $1576.69 \mathrm{de}$ & $1683.81 \mathrm{~cd}$ & $1859.86 \mathrm{ab}$ & $1706.79 \mathrm{~B}$ & $1711.19 \mathrm{de}$ & $1804.15 \mathrm{~cd}$ & $1974.12 \mathrm{ab}$ & $1829.82 \mathrm{~B}$ \\
\hline B $100+$ Mo 50 & $1625.08 \mathrm{de}$ & $1799.74 \mathrm{bc}$ & $1980.17 \mathrm{a}$ & $1801.66 \mathrm{~A}$ & $1758.81 \mathrm{~d}$ & $1929.03 \mathrm{bc}$ & $2099.11 \mathrm{a}$ & $1928.98 \mathrm{~A}$ \\
\hline Mean & $1433.40 \mathrm{C}$ & $1565.43 \mathrm{~B}$ & $1678.93 \mathrm{~A}$ & & $1535.57 \mathrm{C}$ & $1641.92 \mathrm{~B}$ & $1757.14 \mathrm{~A}$ & \\
\hline \multicolumn{9}{|c|}{ Curd diameter $(\mathrm{cm})$} \\
\hline B $0+$ Mo 0 & $18.69 \mathrm{~g}$ & $18.92 \mathrm{~g}$ & $20.16 \mathrm{fg}$ & $19.26 \mathrm{C}$ & $19.26 \mathrm{~g}$ & $19.45 \mathrm{~g}$ & $20.57 \mathrm{fg}$ & $19.76 \mathrm{C}$ \\
\hline B 100 & $20.53 \mathrm{fg}$ & $22.01 \mathrm{ef}$ & $22.52 \mathrm{de}$ & $21.69 \mathrm{~B}$ & $20.60 \mathrm{fg}$ & $22.07 \mathrm{ef}$ & $22.74 \mathrm{e}$ & $21.80 \mathrm{~B}$ \\
\hline Mo 50 & $22.81 \mathrm{de}$ & $25.45 \mathrm{c}$ & $27.45 \mathrm{ab}$ & $25.24 \mathrm{~A}$ & $23.70 \mathrm{de}$ & $25.49 \mathrm{~cd}$ & $27.59 \mathrm{ab}$ & $25.59 \mathrm{~A}$ \\
\hline B $100+$ Mo 50 & $24.37 \mathrm{~cd}$ & $26.21 \mathrm{bc}$ & $28.34 \mathrm{a}$ & $26.30 \mathrm{~A}$ & $25.18 \mathrm{~cd}$ & $26.45 \mathrm{bc}$ & $28.75 \mathrm{a}$ & $26.79 \mathrm{~A}$ \\
\hline Mean & $21.60 \mathrm{C}$ & $23.15 \mathrm{~B}$ & $24.62 \mathrm{~A}$ & & $22.18 \mathrm{C}$ & $23.36 \mathrm{~B}$ & $24.91 \mathrm{~A}$ & \\
\hline \multicolumn{9}{|c|}{ Curd dry matter $(\%)$} \\
\hline B $0+$ Mo 0 & $14.24 \mathrm{k}$ & $14.51 \mathrm{jk}$ & $14.82 \mathrm{ij}$ & $14.52 \mathrm{C}$ & $15.03 \mathrm{j}$ & $15.32 \mathrm{ij}$ & $15.37 \mathrm{ij}$ & $15.24 \mathrm{C}$ \\
\hline B 100 & $15.10 \mathrm{hi}$ & $15.36 \mathrm{gh}$ & $15.67 \mathrm{fg}$ & $15.38 \mathrm{~B}$ & $15.63 \mathrm{hi}$ & $15.95 \mathrm{gh}$ & $16.20 \mathrm{fg}$ & $15.93 \mathrm{~B}$ \\
\hline Mo 50 & $15.97 \mathrm{ef}$ & $16.54 \mathrm{~cd}$ & $17.12 \mathrm{ab}$ & $16.54 \mathrm{~A}$ & $16.49 \mathrm{ef}$ & $17.09 \mathrm{~cd}$ & $17.72 \mathrm{ab}$ & $17.10 \mathrm{~A}$ \\
\hline B $100+$ Mo 50 & $16.25 \mathrm{de}$ & $16.83 \mathrm{bc}$ & $17.35 \mathrm{a}$ & $16.81 \mathrm{~A}$ & $16.80 \mathrm{de}$ & $17.41 \mathrm{bc}$ & $17.95 \mathrm{a}$ & $17.38 \mathrm{~A}$ \\
\hline Mean & $15.39 \mathrm{C}$ & $15.81 \mathrm{~B}$ & $16.24 \mathrm{~A}$ & & $15.99 \mathrm{~B}$ & $16.44 \mathrm{AB}$ & $16.81 \mathrm{~A}$ & \\
\hline \multicolumn{9}{|c|}{ Curds yield (tone/fed.) } \\
\hline B $0+$ Mo 0 & $13.14 \mathrm{f}$ & $13.31 \mathrm{f}$ & $13.43 \mathrm{f}$ & $13.29 \mathrm{D}$ & $13.16 \mathrm{f}$ & $13.84 \mathrm{f}$ & $14.21 \mathrm{ef}$ & $13.73 \mathrm{D}$ \\
\hline B 100 & $14.01 \mathrm{ef}$ & $14.63 \mathrm{ef}$ & $15.94 \mathrm{de}$ & $14.86 \mathrm{C}$ & $14.28 \mathrm{ef}$ & $15.05 \mathrm{ef}$ & $16.45 \mathrm{de}$ & $15.26 \mathrm{C}$ \\
\hline Mo 50 & $16.41 \mathrm{de}$ & $18.92 \mathrm{bc}$ & $21.04 \mathrm{ab}$ & $18.79 \mathrm{~B}$ & $16.61 \mathrm{de}$ & $19.17 \mathrm{bc}$ & $21.53 \mathrm{ab}$ & $19.10 \mathrm{~B}$ \\
\hline B $100+$ Mo 50 & $17.75 \mathrm{~cd}$ & $20.23 \mathrm{bc}$ & $22.74 \mathrm{a}$ & $20.24 \mathrm{~A}$ & $18.48 \mathrm{~cd}$ & $20.32 \mathrm{bc}$ & $22.93 \mathrm{a}$ & $20.58 \mathrm{~A}$ \\
\hline Mean & $15.33 \mathrm{C}$ & $16.77 \mathrm{~B}$ & $18.29 \mathrm{~A}$ & & $15.63 \mathrm{C}$ & $17.09 \mathrm{~B}$ & $18.78 \mathrm{~A}$ & \\
\hline \multicolumn{9}{|c|}{ Days to curd maturity } \\
\hline B $0+$ Mo 0 & $92.54 \mathrm{a}$ & $93.00 \mathrm{a}$ & $93.33 \mathrm{a}$ & $92.96 \mathrm{~A}$ & $94.00 \mathrm{a}$ & $94.00 \mathrm{a}$ & $94.00 \mathrm{a}$ & $94.00 \mathrm{~A}$ \\
\hline B 100 & $91.85 \mathrm{a}$ & $92.33 \mathrm{a}$ & $94.33 \mathrm{a}$ & $92.84 \mathrm{~A}$ & $93.67 \mathrm{a}$ & $94.00 \mathrm{a}$ & $94.00 \mathrm{a}$ & $93.89 \mathrm{~A}$ \\
\hline Mo 50 & $92.00 \mathrm{a}$ & $92.67 \mathrm{a}$ & $94.00 \mathrm{a}$ & $92.89 \mathrm{~A}$ & $93.67 \mathrm{a}$ & $95.00 \mathrm{a}$ & $94.33 \mathrm{a}$ & $94.33 \mathrm{~A}$ \\
\hline B $100+$ Mo 50 & $92.10 \mathrm{a}$ & $92.33 \mathrm{a}$ & $93.33 \mathrm{a}$ & $92.59 \mathrm{~A}$ & $93.33 \mathrm{a}$ & $94.00 \mathrm{a}$ & $94.00 \mathrm{a}$ & $93.78 \mathrm{~A}$ \\
\hline Mean & $92.12 \mathrm{~A}$ & $92.58 \mathrm{~A}$ & $93.75 \mathrm{~A}$ & & $93.67 \mathrm{~A}$ & $94.25 \mathrm{~A}$ & $94.08 \mathrm{~A}$ & \\
\hline
\end{tabular}

Values are the means of three replicates. Values followed by the same letters within a column for each genus are not significantly different at the $1 \%$ level of probability according to Duncan's multiple range test. $\mathrm{N1}=30 \mathrm{~kg}$ nitrogen/fed., $\mathrm{N} 2=45 \mathrm{~kg}$ nitrogen/fed., $\mathrm{N3}=$ $60 \mathrm{~kg}$ nitrogen/fed., B $0+$ Mo $0=$ control, B 100= $100 \mathrm{ppm}$ of boron, Mo 50=50 ppm of molybdenum and B $100+$ Mo $50=100 \mathrm{ppm}$ of boron combined with $50 \mathrm{ppm}$ of molybdenum.

Effect of nitrogen levels as well as $B$ and Mo on quality parameters

Table (4) shows that all quality parameters were significantly increased by increasing the level of $\mathrm{N}$ in both seasons, except total phenols content. In this line, the highest level of nitrogen; $60 \mathrm{~kg} / \mathrm{fed}$. was more effective for enhancing all studied traits, except total phenols content than the other lower levels: 30 and 45 $\mathrm{kg} / \mathrm{fed}$. Table (4) also shows that the response of all cauliflower quality parameters was significantly affected by foliar application of both B and Mo either individually or together. The maximum content of ascorbic acid, carbohydrates, SSC and sulfur were recorded with foliar combined application of B at 100 ppm and Mo at $50 \mathrm{ppm}$, followed by application of B at 100 ppm only. However, the highest content of total phenols and nitrate content were recorded in cauliflower un-treated plants in both growing seasons. Concerning 
the interaction between $\mathrm{N}$ levels and foliar application of micronutrients, data given in Table (4) demonstrate that the high level of $\mathrm{N}(60 \mathrm{~kg} / \mathrm{fed}$.) in combination with the foliar application of B and Mo together at $100 \mathrm{ppm}$ and $50 \mathrm{ppm}$, respectively, achieved the best records for all quality traits except total phenols and nitrate content.

Effect of nitrogen levels as well as $B$ and Mo on chlorophyll content and mineral composition

Data regarding to the main effects of nitrogen level and micronutrient treatments as well as their interaction effect on chlorophyll content and mineral contents (N, P, K, B and Mo) in cauliflower leaves are presented in table (5). The results undoubtedly indicated that the content of chlorophyll and all minerals were significantly affected by nitrogen level and micronutrient application. All studied traits were increased with increasing the nitrogen level up to the highest level (60 kg/Fed.) in both seasons. Also, the content of chlorophyll and all measured minerals was strongly affected by the micronutrient application; whereas, the highest records of total chlorophyll, N, P, $\mathrm{K}, \mathrm{B}$ and Mo were found in sprayed plants with 100 and $50 \mathrm{ppm}$ of $\mathrm{B}$ and Mo together, respectively, in both growing seasons. Table (5) reveals that the interaction treatment between the high level of $\mathrm{N}(60 \mathrm{~kg} / \mathrm{fed})$ combined with the foliar application of B and Mo (100 and $50 \mathrm{ppm}$, respectively) had a significant effect on total chlorophyll, N, P, K, B and Mo concentrations in both seasons.

Table 4. Main effects of nitrogen levels, micronutrients treatments and their interactions on bio-chemical compounds in curds of cauliflower.

\begin{tabular}{|c|c|c|c|c|c|c|c|c|}
\hline \multirow{2}{*}{ Treatments } & \multicolumn{4}{|c|}{ 2017/2018 season } & \multicolumn{4}{|c|}{ 2018/2019 season } \\
\hline & N1 & N2 & N3 & Mean & N1 & N2 & $\mathbf{N 3}$ & Mean \\
\hline \multicolumn{9}{|c|}{ Ascorbic acid (mg/100g F.W) } \\
\hline B $0+\operatorname{Mo} 0$ & $36.13 \mathrm{i}$ & $36.76 \mathrm{hi}$ & $37.59 \mathrm{gh}$ & $36.83 \mathrm{D}$ & $37.63 \mathrm{j}$ & $38.63 \mathrm{ij}$ & $39.16 \mathrm{hi}$ & $38.47 \mathrm{D}$ \\
\hline B 100 & $40.20 \mathrm{de}$ & $40.26 \mathrm{de}$ & $41.53 \mathrm{~cd}$ & $40.66 \mathrm{~B}$ & $41.90 \mathrm{ef}$ & $42.63 \mathrm{de}$ & $43.36 \mathrm{~cd}$ & $42.63 \mathrm{~B}$ \\
\hline Mo 50 & $38.10 \mathrm{gh}$ & $38.80 \mathrm{fg}$ & $39.63 \mathrm{ef}$ & $38.84 \mathrm{C}$ & $39.93 \mathrm{gh}$ & $40.43 \mathrm{gh}$ & $41.10 \mathrm{fg}$ & $40.48 \mathrm{C}$ \\
\hline B $100+$ Mo 50 & $42.33 \mathrm{bc}$ & $43.20 \mathrm{ab}$ & $43.73 \mathrm{a}$ & $43.08 \mathrm{~A}$ & $44.08 \mathrm{bc}$ & $44.83 \mathrm{ab}$ & $45.40 \mathrm{a}$ & $44.77 \mathrm{~A}$ \\
\hline Mean & $39.19 \mathrm{~B}$ & $39.75 \mathrm{~B}$ & $40.62 \mathrm{~A}$ & & $40.88 \mathrm{C}$ & $41.63 \mathrm{~B}$ & $42.25 \mathrm{~A}$ & \\
\hline \multicolumn{9}{|c|}{ Carbohydrate $(\%)$} \\
\hline $\mathrm{B} 0+\mathrm{Mo} 0$ & $13.54 \mathrm{~h}$ & $13.72 \mathrm{gh}$ & $13.81 \mathrm{gh}$ & $13.69 \mathrm{D}$ & $13.84 \mathrm{i}$ & $14.02 \mathrm{hi}$ & $14.18 \mathrm{gh}$ & $14.01 \mathrm{D}$ \\
\hline B 100 & $14.43 \mathrm{de}$ & $14.56 \mathrm{~cd}$ & $14.75 \mathrm{bc}$ & $14.58 \mathrm{~B}$ & $14.75 \mathrm{de}$ & $14.84 \mathrm{~cd}$ & $14.97 \mathrm{~cd}$ & $14.85 \mathrm{~B}$ \\
\hline Mo 50 & $13.96 \mathrm{fg}$ & $14.14 \mathrm{ef}$ & $14.27 \mathrm{de}$ & $14.12 \mathrm{C}$ & $14.23 \mathrm{gh}$ & $14.38 \mathrm{fg}$ & $14.54 \mathrm{ef}$ & $14.38 \mathrm{C}$ \\
\hline B $100+$ Mo 50 & $14.86 \mathrm{bc}$ & $15.02 \mathrm{ab}$ & $15.32 \mathrm{a}$ & $15.07 \mathrm{~A}$ & $15.15 \mathrm{bc}$ & $15.29 \mathrm{ab}$ & $15.46 \mathrm{a}$ & $15.30 \mathrm{~A}$ \\
\hline Mean & $14.20 \mathrm{~B}$ & $14.36 \mathrm{AB}$ & $14.54 \mathrm{~A}$ & & $14.49 \mathrm{~B}$ & $14.63 \mathrm{AB}$ & $14.78 \mathrm{~A}$ & \\
\hline \multicolumn{9}{|c|}{ Total phenols (mg/100g F.W) } \\
\hline B $0+\operatorname{Mo} 0$ & $397.00 \mathrm{a}$ & $391.33 \mathrm{ab}$ & $386.66 \mathrm{bc}$ & $391.66 \mathrm{~A}$ & $376.66 \mathrm{a}$ & $370.33 \mathrm{ab}$ & $363.66 \mathrm{bc}$ & $370.22 \mathrm{~A}$ \\
\hline B 100 & $362.33 \mathrm{ef}$ & $353.66 \mathrm{fg}$ & $351.33 \mathrm{gh}$ & $355.77 \mathrm{C}$ & $347.00 \mathrm{ef}$ & $342.33 \mathrm{fg}$ & $339.00 \mathrm{gh}$ & $342.77 \mathrm{C}$ \\
\hline Mo 50 & $382.33 \mathrm{bc}$ & $378.66 \mathrm{~cd}$ & $372.00 \mathrm{de}$ & $377.66 \mathrm{~B}$ & $358.33 \mathrm{~cd}$ & $352.33 \mathrm{de}$ & $349.33 \mathrm{ef}$ & $353.33 \mathrm{~B}$ \\
\hline B $100+$ Mo 50 & $348.33 \mathrm{gh}$ & $341.66 \mathrm{hi}$ & $335.33 \mathrm{i}$ & $341.77 \mathrm{D}$ & $332.00 \mathrm{~h}$ & $322.66 \mathrm{i}$ & $318.33 \mathrm{i}$ & $324.33 \mathrm{D}$ \\
\hline Mean & $372.50 \mathrm{~A}$ & $366.33 \mathrm{AB}$ & $361.33 \mathrm{~B}$ & & $353.50 \mathrm{~A}$ & $346.91 \mathrm{~B}$ & $342.58 \mathrm{~B}$ & \\
\hline \multicolumn{9}{|c|}{$\mathrm{SSC}(\%)$} \\
\hline $\mathrm{B} 0+\mathrm{Mo} 0$ & $6.76 \mathrm{f}$ & $6.85 \mathrm{f}$ & 6.94 ef & $6.85 \mathrm{D}$ & $6.78 \mathrm{i}$ & $6.86 \mathrm{hi}$ & $6.97 \mathrm{hi}$ & $6.87 \mathrm{D}$ \\
\hline B 100 & $7.29 \mathrm{~cd}$ & $7.37 \mathrm{~cd}$ & $7.46 \mathrm{bc}$ & $7.37 \mathrm{~B}$ & 7.36 ef & $7.43 \mathrm{de}$ & $7.58 \mathrm{~cd}$ & $7.45 \mathrm{~B}$ \\
\hline Mo 50 & $6.98 \mathrm{ef}$ & $7.12 \mathrm{de}$ & $7.19 \mathrm{de}$ & $7.10 \mathrm{C}$ & $7.06 \mathrm{gh}$ & $7.19 \mathrm{fg}$ & 7.28 ef & $7.18 \mathrm{C}$ \\
\hline B $100+$ Mo 50 & $7.51 \mathrm{bc}$ & $7.65 \mathrm{ab}$ & $7.77 \mathrm{a}$ & $7.64 \mathrm{~A}$ & $7.64 \mathrm{bc}$ & $7.82 \mathrm{ab}$ & $7.92 \mathrm{a}$ & $7.79 \mathrm{~A}$ \\
\hline Mean & $7.14 \mathrm{C}$ & $7.24 \mathrm{~B}$ & $7.34 \mathrm{~A}$ & & $7.21 \mathrm{C}$ & $7.32 \mathrm{~B}$ & $7.44 \mathrm{~A}$ & \\
\hline \multicolumn{9}{|c|}{$\mathrm{NO}_{3}-\mathrm{N}(\mathrm{ppm})$} \\
\hline B $0+$ Mo 0 & $134.20 \mathrm{bc}$ & $139.70 \mathrm{ab}$ & $143.33 \mathrm{a}$ & $139.07 \mathrm{~A}$ & $136.63 \mathrm{bc}$ & $142.50 \mathrm{ab}$ & $144.13 \mathrm{a}$ & $141.08 \mathrm{~A}$ \\
\hline B 100 & $118.06 \mathrm{ef}$ & $123.53 \mathrm{de}$ & $129.20 \mathrm{~cd}$ & $123.60 \mathrm{~B}$ & $128.16 \mathrm{~d}$ & $131.50 \mathrm{~cd}$ & $133.60 \mathrm{~cd}$ & $131.08 \mathrm{~B}$ \\
\hline Mo 50 & $103.80 \mathrm{hi}$ & $108.53 \mathrm{gh}$ & $113.13 \mathrm{fg}$ & $108.48 \mathrm{C}$ & $105.63 \mathrm{fg}$ & $109.46 \mathrm{ef}$ & $114.20 \mathrm{e}$ & $109.76 \mathrm{C}$ \\
\hline B $100+$ Mo 50 & $89.26 \mathrm{j}$ & $94.80 \mathrm{ij}$ & $99.26 \mathrm{i}^{\circ}$ & $94.44 \mathrm{D}$ & $94.56 \mathrm{~h}$ & $99.46 \mathrm{gh}$ & $103.40 \mathrm{fg}$ & $99.14 \mathrm{D}$ \\
\hline Mean & $111.33 \mathrm{~B}$ & $116.64 \mathrm{AB}$ & $121.23 \mathrm{~A}$ & & $116.25 \mathrm{~B}$ & $120.73 \mathrm{AB}$ & $123.83 \mathrm{~A}$ & \\
\hline \multicolumn{9}{|c|}{$\mathrm{S}(\mathrm{ppm})$} \\
\hline $\mathrm{B} 0+\mathrm{Mo} 0$ & $0.076 \mathrm{i}$ & $0.088 \mathrm{hi}$ & $0.097 \mathrm{~h}$ & $0.087 \mathrm{D}$ & $0.077 \mathrm{~g}$ & $0.096 \mathrm{fg}$ & $0.109 \mathrm{f}$ & $0.094 \mathrm{D}$ \\
\hline B 100 & $0.142 \mathrm{e}$ & $0.153 \mathrm{de}$ & $0.164 \mathrm{~cd}$ & $0.153 \mathrm{~B}$ & $0.143 \mathrm{de}$ & $0.163 \mathrm{~cd}$ & $0.172 \mathrm{c}$ & $0.159 \mathrm{~B}$ \\
\hline Mo 50 & $0.104 \mathrm{gh}$ & $0.119 \mathrm{fg}$ & $0.134 \mathrm{ef}$ & $0.119 \mathrm{C}$ & $0.109 \mathrm{f}$ & $0.131 \mathrm{e}$ & $0.142 \mathrm{de}$ & $0.127 \mathrm{C}$ \\
\hline B $100+$ Mo 50 & $0.176 \mathrm{bc}$ & $0.187 \mathrm{ab}$ & $0.197 \mathrm{a}$ & $0.187 \mathrm{~A}$ & $0.177 \mathrm{bc}$ & $0.194 \mathrm{~b}$ & $0.259 \mathrm{a}$ & $0.210 \mathrm{~A}$ \\
\hline Mean & $0.124 \mathrm{C}$ & $0.137 \mathrm{~B}$ & $0.148 \mathrm{~A}$ & & $0.126 \mathrm{~B}$ & $0.146 \mathrm{AB}$ & $0.171 \mathrm{~A}$ & \\
\hline
\end{tabular}


Table 5. Main effects of nitrogen levels, micronutrients treatments and their interactions on total chlorophyll, macronutrients and micronutrients in leaves of cauliflower.

\begin{tabular}{|c|c|c|c|c|c|c|c|c|}
\hline \multirow{2}{*}{ Treatments } & \multicolumn{4}{|c|}{ 2017/2018 season } & \multicolumn{4}{|c|}{ 2018/2019 season } \\
\hline & N1 & $\mathbf{N 2}$ & $\mathbf{N 3}$ & Mean & N1 & $\mathbf{N 2}$ & $\mathbf{N 3}$ & Mean \\
\hline \multicolumn{9}{|c|}{ Total chlorophyll (mg/g F.W) } \\
\hline$\overline{\mathrm{B} 0+\mathrm{Mo} 0}$ & $0.904 \mathrm{~h}$ & $0.931 \mathrm{~g}$ & $0.946 \mathrm{fg}$ & $0.927 \mathrm{D}$ & $0.948 \mathrm{j}$ & $0.971 \mathrm{ij}$ & $0.982 \mathrm{hi}$ & $0.967 \mathrm{D}$ \\
\hline B 100 & $1.024 \mathrm{~d}$ & $1.047 \mathrm{c}$ & $1.060 \mathrm{c}$ & $1.044 \mathrm{~B}$ & $1.052 \mathrm{de}$ & $1.069 \mathrm{~cd}$ & $1.084 \mathrm{c}$ & $1.068 \mathrm{~B}$ \\
\hline Mo 50 & 0.963 ef & $0.983 \mathrm{e}$ & $1.007 \mathrm{~d}$ & $0.984 \mathrm{C}$ & $1.001 \mathrm{gh}$ & $1.019 \mathrm{fg}$ & $1.035 \mathrm{ef}$ & $1.018 \mathrm{C}$ \\
\hline B $100+$ Mo 50 & $1.088 \mathrm{~b}$ & $1.107 \mathrm{ab}$ & $1.127 \mathrm{a}$ & $1.107 \mathrm{~A}$ & $1.094 \mathrm{bc}$ & $1.118 \mathrm{ab}$ & $1.136 \mathrm{a}$ & $1.116 \mathrm{~A}$ \\
\hline Mean & $0.995 \mathrm{C}$ & $1.017 \mathrm{~B}$ & $1.035 \mathrm{~A}$ & & $1.023 \mathrm{~B}$ & $1.044 \mathrm{~A}$ & $1.059 \mathrm{~A}$ & \\
\hline \multicolumn{9}{|c|}{$\mathrm{N}(\%)$} \\
\hline$\overline{\mathrm{B} 0+\mathrm{Mo} 0}$ & $0.85 \mathrm{j}$ & $0.90 \mathrm{j}$ & $0.98 \mathrm{ij}$ & $0.91 \mathrm{D}$ & $0.98 \mathrm{~g}$ & $1.05 \mathrm{~g}$ & $1.13 \mathrm{fg}$ & $1.05 \mathrm{D}$ \\
\hline В 100 & $1.27 \mathrm{ef}$ & $1.38 \mathrm{de}$ & $1.44 \mathrm{~cd}$ & $1.36 \mathrm{~B}$ & $1.46 \mathrm{~d}$ & $1.51 \mathrm{~cd}$ & $1.62 \mathrm{bc}$ & $1.53 \mathrm{~B}$ \\
\hline Mo 50 & $1.04 \mathrm{hi}$ & $1.13 \mathrm{gh}$ & $1.21 \mathrm{fg}$ & $1.13 \mathrm{C}$ & $1.20 \mathrm{f}$ & $1.28 \mathrm{ef}$ & $1.36 \mathrm{de}$ & $1.28 \mathrm{C}$ \\
\hline B $100+$ Mo 50 & $1.53 \mathrm{bc}$ & $1.62 \mathrm{ab}$ & $1.67 \mathrm{a}$ & $1.61 \mathrm{~A}$ & $1.68 \mathrm{~b}$ & $1.75 \mathrm{ab}$ & $1.84 \mathrm{a}$ & $1.76 \mathrm{~A}$ \\
\hline Mean & $1.17 \mathrm{~B}$ & $1.26 \mathrm{AB}$ & $1.33 \mathrm{~A}$ & & $1.33 \mathrm{~B}$ & $1.40 \mathrm{AB}$ & $1.49 \mathrm{~A}$ & \\
\hline \multicolumn{9}{|c|}{$\mathrm{P}(\%)$} \\
\hline$\overline{\mathrm{B} 0+\mathrm{Mo} 0}$ & $0.102 \mathrm{i}$ & $0.110 \mathrm{hi}$ & $0.122 \mathrm{gh}$ & $0.111 \mathrm{D}$ & $0.120 \mathrm{~g}$ & $0.128 \mathrm{~g}$ & $0.138 \mathrm{fg}$ & $0.129 \mathrm{D}$ \\
\hline В 100 & $0.153 \mathrm{de}$ & $0.159 \mathrm{~cd}$ & $0.167 \mathrm{~cd}$ & $0.160 \mathrm{~B}$ & $0.176 \mathrm{~cd}$ & $0.180 \mathrm{~cd}$ & $0.192 \mathrm{bc}$ & $0.183 \mathrm{~B}$ \\
\hline Mo 50 & $0.128 \mathrm{fg}$ & $0.135 \mathrm{fg}$ & 0.142 ef & $0.135 \mathrm{C}$ & 0.148 ef & $0.158 \mathrm{e}$ & $0.167 \mathrm{de}$ & $0.157 \mathrm{C}$ \\
\hline B $100+$ Mo 50 & $0.171 b c$ & $0.187 \mathrm{ab}$ & $0.192 \mathrm{a}$ & $0.183 \mathrm{~A}$ & $0.200 \mathrm{~b}$ & $0.208 \mathrm{ab}$ & $0.219 \mathrm{a}$ & $0.209 \mathrm{~A}$ \\
\hline Mean & $0.138 \mathrm{C}$ & $0.148 \mathrm{~B}$ & $0.155 \mathrm{~A}$ & & $0.161 \mathrm{~B}$ & $0.168 \mathrm{AB}$ & $0.179 \mathrm{~A}$ & \\
\hline \multicolumn{9}{|c|}{$\mathrm{K}(\%)$} \\
\hline $\mathrm{B} 0+\mathrm{Mo} 0$ & $1.25 \mathrm{j}$ & $1.31 \mathrm{ij}$ & $1.40 \mathrm{hi}$ & $1.32 \mathrm{D}$ & $1.26 \mathrm{~h}$ & $1.39 \mathrm{gh}$ & $1.41 \mathrm{gh}$ & $1.35 \mathrm{D}$ \\
\hline В 100 & $1.58 \mathrm{ef}$ & $1.65 \mathrm{de}$ & $1.72 \mathrm{~cd}$ & $1.65 \mathrm{~B}$ & $1.77 \mathrm{de}$ & $1.86 \mathrm{~cd}$ & $1.93 \mathrm{~cd}$ & $1.85 \mathrm{~B}$ \\
\hline Mo 50 & $1.46 \mathrm{gh}$ & $1.48 \mathrm{gh}$ & $1.53 \mathrm{fg}$ & $1.49 \mathrm{C}$ & $1.51 \mathrm{fg}$ & $1.58 \mathrm{f}$ & $1.67 \mathrm{ef}$ & $1.58 \mathrm{C}$ \\
\hline B $100+$ Mo 50 & $1.81 \mathrm{bc}$ & $1.88 \mathrm{ab}$ & $1.98 \mathrm{a}$ & $1.89 \mathrm{~A}$ & $2.02 \mathrm{bc}$ & $2.10 \mathrm{ab}$ & $2.19 \mathrm{a}$ & $2.10 \mathrm{~A}$ \\
\hline Mean & $1.52 \mathrm{~B}$ & $1.58 \mathrm{~B}$ & $1.65 \mathrm{~A}$ & & $1.64 \mathrm{~B}$ & $1.73 \mathrm{~A}$ & $1.80 \mathrm{~A}$ & \\
\hline \multicolumn{9}{|c|}{$\mathrm{B}(\mathrm{ppm})$} \\
\hline $\mathrm{B} 0+\mathrm{Mo} 0$ & $33.70 \mathrm{~h}$ & $34.80 \mathrm{gh}$ & $36.13 \mathrm{fg}$ & $34.87 \mathrm{C}$ & $35.56 \mathrm{i}$ & $36.86 \mathrm{hi}$ & $37.86 \mathrm{hi}$ & $36.76 \mathrm{C}$ \\
\hline В 100 & $40.53 \mathrm{~cd}$ & $42.73 b c$ & $44.80 \mathrm{ab}$ & $42.68 \mathrm{~A}$ & $42.70 \mathrm{de}$ & $45.10 \mathrm{bc}$ & $47.36 \mathrm{ab}$ & $45.05 \mathrm{~A}$ \\
\hline Mo 50 & $37.26 \mathrm{ef}$ & 38.16 ef & $39.26 \mathrm{de}$ & $38.23 \mathrm{~B}$ & $39.10 \mathrm{gh}$ & $40.23 \mathrm{fg}$ & 41.46 ef & $40.26 \mathrm{~B}$ \\
\hline B $100+$ Mo 50 & $41.53 \mathrm{c}$ & $44.00 \mathrm{~b}$ & $46.33 \mathrm{a}$ & $43.95 \mathrm{~A}$ & $43.86 \mathrm{~cd}$ & $46.20 \mathrm{~b}$ & $48.73 \mathrm{a}$ & $46.26 \mathrm{~A}$ \\
\hline Mean & $38.25 \mathrm{C}$ & $39.92 \mathrm{~B}$ & $41.63 \mathrm{~A}$ & & $40.30 \mathrm{C}$ & $42.10 \mathrm{~B}$ & $43.85 \mathrm{~A}$ & \\
\hline \multicolumn{9}{|c|}{ Mo (ppm) } \\
\hline B $0+$ Mo 0 & $0.68 \mathrm{i}$ & $0.85 \mathrm{i}$ & $1.08 \mathrm{hi}$ & $0.87 \mathrm{C}$ & $0.88 \mathrm{~h}$ & $1.04 \mathrm{gh}$ & $1.25 \mathrm{gh}$ & $1.06 \mathrm{C}$ \\
\hline В 100 & $1.36 \mathrm{gh}$ & $1.63 \mathrm{fg}$ & $1.90 \mathrm{ef}$ & $1.63 \mathrm{~B}$ & $1.47 \mathrm{fg}$ & $1.74 \mathrm{ef}$ & $1.97 \mathrm{de}$ & $1.72 \mathrm{~B}$ \\
\hline Mo 50 & $2.14 \mathrm{de}$ & $2.61 \mathrm{bc}$ & $3.05 \mathrm{ab}$ & $2.60 \mathrm{~A}$ & $2.17 \mathrm{de}$ & $2.64 \mathrm{bc}$ & $3.09 \mathrm{ab}$ & $2.63 \mathrm{~A}$ \\
\hline B $100+$ Mo 50 & $2.40 \mathrm{~cd}$ & $2.82 \mathrm{bc}$ & $3.31 \mathrm{a}$ & $2.84 \mathrm{~A}$ & $2.41 \mathrm{~cd}$ & $2.86 \mathrm{bc}$ & $3.39 \mathrm{a}$ & $2.88 \mathrm{~A}$ \\
\hline Mean & $1.64 \mathrm{~B}$ & $1.98 \mathrm{~B}$ & $2.33 \mathrm{~A}$ & & $1.73 \mathrm{C}$ & $2.07 \mathrm{~B}$ & $2.42 \mathrm{~A}$ & \\
\hline
\end{tabular}

Values are the means of three replicates. Values followed by the same letters within a column for each genus are not significantly different at the $1 \%$ level of probability according to Duncan's multiple range test. N1=30 kg nitrogen/fed., N2=45 kg nitrogen/fed., N3= $60 \mathrm{~kg}$ nitrogen/fed., B $0+$ Mo $0=$ control, B 100 $=100 \mathrm{ppm}$ of boron, Mo 50=50 ppm of molybdenum and B $100+$ Mo $50=100 \mathrm{ppm}$ of boron combined with $50 \mathrm{ppm}$ of molybdenum.

\section{Discussion}

Cauliflower is one the vegetable crops that need nitrogen in optimum level, because inadequate dose of nitrogen may lead to a decay in curd formation and quality parameters (Bianco et al., 2015). Therefore, the current research was primarily performed out to study the effect of different levels of $\mathrm{N}(30,45$, and $60 \mathrm{~kg} / \mathrm{fed}$.) to determine the optimum level of $\mathrm{N}$ in newly reclaimed areas, especially New Valley governorate as a promising area for agricultural expansion in Egypt. The results of this research reported that all vegetative traits; plant height, number of leaves, leaf area, fresh weigh of leaves and dry matter of leaves gradually increased with the increasing level of N. Similarly, it has been previously reported that vegetative growth parameters of cauliflower were significantly influenced by different $\mathrm{N}$ fertilization (Farahzety and Siti Aishah, 2013). Also, our results show that all yield traits were increased linearly with increasing level of mineral $\mathrm{N}$ up to $60 \mathrm{~kg} / \mathrm{fed}$. except number of days to maturity. Similar results were obtained by previous reports, which declared that curd weight, diameter and dry matter were generally responsive to mineral $\mathrm{N}$ application (Farrag et al., 2000; Abdel-Razzak et al., 2008 a and b).

Moreover, our study clearly show that all quality parameters: vitamin C, carbohydrates, soluble solids content, nitrate and sulfur content were increased with increasing the inorganic $\mathrm{N}$ level with exception of total phenols. In this direction, Abdel-Razzak et al. (2008 b) reported that inorganic $\mathrm{N}$ has a great influence on cauliflower curd quality related traits.

Generally, this study clearly confirmed that nearly all vegetative growth, yield and quality traits showed significant differences due to $\mathrm{N}$ application in both growing seasons; whereas the highest level of $N(60 \mathrm{~kg} / \mathrm{fed}$.) gave the maximum records of most of studied traits in the current study (Table 2, 3, and 4). This promotional effect of $\mathrm{N}$ in enhancing the growth, yield and quality of cauliflower might be explained by the fact that $\mathrm{N}$ is one of the most critical constitutes for all biochemical and physiological functions that are running during the cauliflower growth. For instance, $\mathrm{N}$ is an essential constitute in synthesis of chlorophyll, amino acids, protein, nucleic acids and hormones, which might rabidly enhance the synthesized carbohydrates that critical for more dry matter accumulation in plant organs (Russel, 1973; Leghari et al., 2016). In our study, nitrogen application had a positive effect on the total chlorophyll content (Table 5); whereas, the $\mathrm{N}$ fertilized plants with the highest level (60 $\mathrm{kg} / \mathrm{fed}$.) had recorded the highest total chlorophyll content (1.035 and $1.059 \mathrm{mg} / \mathrm{g} \mathrm{FW}$ ) and carbohydrates content (14.54 and $14.78 \%$ ) in the first and second seasons, respectively. In the same regard, Mitra et al. (1990) reported 
that chlorophyll content was increases by increasing $\mathrm{N}$ application from 56 to $224 \mathrm{~kg} / \mathrm{ha}$ in broccoli. In addition, the observed nitrogen promotional effect in this study might be due to the role of $\mathrm{N}$ in improving the mineral content of macronutrients and micronutrients in leaves of cauliflower as shown in Table (5). Where, the highest content of macronutrients $(\mathrm{N}, \mathrm{P}$ and $\mathrm{K})$ and micronutrients $(\mathrm{B}$ and $\mathrm{Mo})$ were recorded in leaves of treated plants with $60 \mathrm{~kg} / \mathrm{fed}$. Similarly, inorganic and organic $\mathrm{N}$ application have improved of cauliflower curds of macronutrients and micronutrients. Together, the positive effect of mineral $\mathrm{N}$ application on mineral contents of cauliflower appeared to enhance cauliflower growth by enhancing photosynthetic activities as well as minerals uptake and availability, which ultimately leads to more assimilation of carbohydrates and their translocation to the reproductive tissues, namely curds (Sharma et al., 2002).

Besides N, micronutrients application also play an important role in plant nutrition, particularly cauliflower. Therefore, another aim of current study was to find out necessity of micronutrient, $\mathrm{B}$ and $\mathrm{Mo}$, in cauliflower cultivation. Generally, the results showed positive effects of using either B or Mo on cauliflower curds yield and quality characters compared with untreated plants. However, combined foliar application of $100 \mathrm{ppm}$ of B and $50 \mathrm{ppm}$ of Mo recorded the highest values of vegetative, yield and quality parameters. Similarly, several recent studies confirmed that application of B and Mo strongly affected the growth, yield and quality of cauliflower (Thapa et al., 2016; Hossain et al., 2018; Sarker et al., 2018, Hassan et al., 2018). Micronutrients deficiency often occur in plants grown in sandy soil and new reclaimed areas (Shaaban, 2010). However, plant crop species greatly vary in their demand to micronutrients. Cauliflower is sensitive to $\mathrm{B}$ and $\mathrm{Mo}$ and it often shows B and Mo deficiency. The promotional effects of $\mathrm{B}$ and Mo might be referred to their critical roles in synthesis and metabolism of carbohydrates as well as enhancing photosynthetic process, where Tables 4 and 5 show the combined application of $\mathrm{B}$ and Mo recorded the maximum values of carbohydrates content and total chlorophyll in the first $(15.07 \%$ and $1.107 \mathrm{mg} \mathrm{g} / \mathrm{FW})$ and second seasons (15.30 \% and $1.116 \mathrm{mg}$ g/FW). In this regards, Lewis (1980) reported that $\mathrm{B}$ controls the metabolic reactions of carbohydrates by controlling several enzyme activities, such as $\alpha$ and $\beta$ amylase. Also, Ahmed et al. (2014) stated a positive effect for external $\mathrm{B}$ addition on chlorophyll content in cotton plants.

Excessive nitrate is harmful for human health, particularly for children and patients nutrition (Makovic and Djurovka, 1990); therefore, it is considered an important characteristic to be monitored in agricultural products in response to nitrogen fertilization. As expected, the highest level of nitrate content was recorded in plant treated with inorganic $\mathrm{N}$ at $60 \mathrm{~kg} / \mathrm{fed}$. with a maximum of 121.23 and $123.83 \mathrm{ppm}$ in the first and second seasons, respectively, which is still in the safe level for human consumption. The same result was indicated by Abdel-Razzak et al. (2008 b), who found that the content of nitrate in cauliflower head was increased by increasing $\mathrm{N}$ fertilization dose from 40 to $120 \mathrm{~kg}$ $\mathrm{N} /$ fed. Nevertheless, the application of $\mathrm{B}$ and Mo significantly decreased the nitrate content in cauliflower plants over the three levels of $\mathrm{N}$ (Table 4). The highest reduction in nitrate content was achieved by foliar spray of combined B and Mo, followed by Mo only. The reduction in nitrate content in this study might be explored by the role of Mo in controlling the activity of nitrate reductase that converts the nitrate to nitrite, which is the first step of the integration of nitrogen to proteins (Kaiser et al., 2005; Bambara and Ndakidemi, 2010). These results confirm the role of $\mathrm{B}$ and $\mathrm{Mo}$ in improving quality parameters in cauliflower curds through decreasing the nitrate content and increasing other quality parameters, such as vitamin C, carbohydrate and SSC.

Interestingly, neither nitrogen levels nor micronutrient application had altered the trait of number days to maturity (Table 2) over the two growing seasons. Contrary, Singh et al. (2017) reported that soil application of Borax (20 kg/ha) combined with Sodium molybdate $(2 \mathrm{~kg} / \mathrm{ha})$ as sources of B and Mo, respectively, accelerated the early mature yield of cauliflower. However, insignificant effects of nitrogen, micronutrient application and their interaction on number of days to maturity might be due to its high heritability, whereas, it was reported that number of days to budding is a highly heritable trait (86 and 94\%), which indicates that this trait is stable and not strongly affected by environmental factors (Yousef et al., 2015; Thorwarth et al., 2018).

Increasing the total phenolic is an advantage for human health, where those phytochemicals possess many biologically significant functions, such as protection against oxidative stress, and degenerative diseases (Forni et al., 2019). Another interesting finding of this research is the insignificant effect of $\mathrm{N}$ and micronutrients application on the phenolic compounds in cauliflower curds. In crux, both $\mathrm{N}$ and micronutrients treatments had negative effect on total phenols content in this study, which in contradiction to results of Abdel-Razzak et al. (2008 b), who reported that addition of different levels of inorganic $\mathrm{N}$ fertilizer caused gradual increase in phenolic content of cauliflower curds. However, the high concentration of total phenols in control plants compared with treated plants may indicate that these plants were subjected to nutrient deficiency, which leads to an increase in the activity of phenylalanine ammonialyase (PAL), leading to a significant increase in total phenols in the stressed plants (Chishaki and Horiguchi, 1997; Liakopoulos and Karabourniotis, 2005).

\section{CONCLUSION}

From the current study, it was stated that for the better production value of cauliflower, the soil application of high nitrogen and foliar spray of B and Mo are necessarily needed and the results confirmed that application of $\mathrm{N}$ at $60 \mathrm{~kg} / \mathrm{fed}$. as well as foliar spray of B and Mo at $100 \mathrm{ppm}$ and $50 \mathrm{ppm}$, respectively are optimum for effective yield in newly reclaimed area in the south-west part of Egypt. Briefly, the application of $\mathrm{N}$ at $60 \mathrm{~kg} / \mathrm{fed}$. combined foliar application of $\mathrm{B}$ and $\mathrm{Mo}$ at $100 \mathrm{ppm}$ and $50 \mathrm{ppm}$, respectively, improved the growth and yield of cauliflower plants grown in sandy soils through enhancing the chlorophyll and carbohydrate content; while they increase the cauliflower quality through reducing the nitrate content.

\section{REFERENCES}

A.O.A.C. Association of Official Analytical Chemists (1975). Official Methods of Analysis, $12^{\text {th }}$ ed. A.O.A.C, Washington DC., USA.

Abdel-All, H. M. and R. A. El-Shabrawy (2013). Effect of some phosphorus sources, nitrogen and sulphur levels on yield, quality, sulforaphane and vit. $\mathrm{C}$ content in broccoli. Res. J. Agri. and Bio. Sci., 9(6): 351-365. 
Abdel-Razzak, H. S., T. H. Solieman and T. H. Gamel (2008 a). Influences of mineral nitrogen fertilizer sources and levels on vegetative growth, yield, curd quality and nutritional value of cauliflower (Brassica oleraceae var. botrytis, L.). Alex. J. Agric. Res., 53(2): 71-84.

Abdel-Razzak, H. S., T. H. Gamel and A. B. El-Nasharty $(2008$ b). Efficiency of inorganic and organic nitrogen fertilization on cauliflower (Brassica oleraceae var. botrytis L.) curds quality. Alex. Sci. Exch. J., 29(4): 283-297.

Agerbirk, N., M. De Vos, J. H. Kim and G. Jander (2009). Indole glucosinolate breakdown and its biological effects. Phytochem. Rev., 8(1): 101.

Ahmed, M. E. S., A. A. Elzaawely and M. B. El-Sawy (2011). Effect of the foliar spraying with molybdenum and magnesium on vegetative growth and curd yields in cauliflower (Brassica oleraceae var. botrytis L.). World J. Agr. Sci., 7(2): 149-156.

Ahmed, N., M. Abid, A. Rashid, R. Abou-Shanab and F. Ahmad (2014). Influence of boron nutrition on membrane leakage, chlorophyll content and gas exchange characteristics in cotton (Gossypium hirsutum L.). J. plant nutr., 37(14): 2302-2315.

Amtmann, A. and P. Armengaud (2009). Effects of N, P, K and $\mathrm{S}$ on metabolism: new knowledge gained from multi-level analysis. Curr. Opin. Plant Bio., 12:275283.

Baethgen, W. E. and M. M. Alley (1989). A manual colorimetric procedure for measuring ammonium nitrogen in soil and plant kjeldahl digests. Comm. Soil Sci. Plant Anal. 20 (9\&10): 961-969.

Bambara, S. and P. A Ndakidemi (2010). The potential roles of lime and molybdenum on the growth, nitrogen fixation and assimilation of matabolites in nodulated legume: A special reference to Phaseolus vulgaris. Afr. J. Bio., (8): 2482-2489.

Bianco, M. S., A. B. Cecílio Filho and L. B. de Carvalho (2015). Nutritional status of the cauliflower cultivar 'Verona'grown with omission of out added macronutrients. PloS one, 10(4): $\mathrm{e} 0123500$.

Camacho-Cristóbal, J. J., M. T. Navarro-Gochicoa, J. Rexach, A. González-Fontes and M. B. HerreraRodríguez (2018). Plant Response to Boron Deficiency and Boron Use Efficiency in Crop Plants. Plant Micronutrient Use Efficiency, 109-121.

Cataldo, D. A., M. Haroon, L. E. Schrader and V. L. Younges (1975). Rapid colorimetric determination of nitrate in plant tissue by nutrition of salicylic acid. Comm. Soil Sci. Plant Anal., 6 (1): 71-80.

Chapman, H. D. and P. F. Pratt (1978). Methods of Analysis for Soils, Plants and Water. Division of Agriculture Sciences, University of California, Davis, pp. 162-165.

Chishaki, N. and T. Horiguchi. (1997). Responses of secondary metabolism to nutrient deficiency. Soil Sci. Plant Nutr., 43:987-991.

Collins, M. and J. E. McCoy (1997). Chicory production, forage quality, and response to nitrogen fertilization. Agron. J., 89: 232-238.

Dible, W. T., E. Truog and K. C. Berger (1954). Boron Determination in Soils and Plants. Anal. Chem., 26(2): 418-421.

Elia, A., P. Santamaria and F. Serio (1998). Nitrogen nutrition, yield and quality of spinach. J. Sci. Food Agric., 76: 341-346.
Elwan, M. W. M. and R. S. A. El-Shatoury (2012). Salicylic acid positively affected plant growth, photosynthetic leaf pigments and fruit yield of summer squash (Cucurbita pepo L.) grown under different n-levels. J. Plant Prod., Mansoura Univ., 3 (7): 2123 - 2138.

Farahzety, A. M. and H. S. Aishah (2013). Effects of organic fertilizers on performance of cauliflower (Brassica oleracea var. botrytis) grown under protected structure. J. Trop. Agric. food. Sci., 41(1): 15-25.

Farooq, M., M. Bakhtiar, S. Ahmed, N. Ilyas, I. Khan, A. Saboor and I. Khan (2018). Influence of Sulfur and Boron on the growth and yield of Broccoli. Inter. J. Env. Agri. Res., 4(4): 9-16.

Farrag, A. M., J. F. Mishriky and A. M. El-Nagar. (2000). Effect of drip irrigation levels and nitrogen rates on growth and yield of cauliflower plants \& salts accumulation in soil. J. Agric. Sci. Mansoura Univ., 25(5): 2855-2875.

Forni, C., F. Facchiano, M. Bartoli, S. Pieretti, A. Facchiano, D. D'Arcangelo and C. Tabolacci (2019). Beneficial Role of Phytochemicals on Oxidative Stress and AgeRelated Diseases. BioMed Res. Inter., 8748253.

Hassan, M. R., N. J. Shamema, A. Ali, K. K. Papon and S. Z. Md (2018). Influence of micronutrient (boron) for the growth and yield of cauliflower. J. Biosci. Agric., Res. 18 (1): 1464-1469.

He-xi, Z., C. Dao-cai, W. Qun, F. Jun and F. Xiao-yu (2011). Yield and quality response of cucumber to irrigation and nitrogen fertilization under subsurface drip irrigation in solar greenhouse. Agri. Sci. China 10(6): 921-930.

Hossain M. F., M. R. Uddi, M. R. Humauan and J. Hossain (2018). Effect of molybdenum and its application on seed production of cauliflower. Bulletin of the Institute of Tropical Agri., Kyushu Uni., 41: 67-72.

Jackson, M. L. (1973). Soil Chemical Analysis. Prentice-Hall of India Private Limited New Delhi, 141 p.

Johnson, C. M., T. H. Arkley (1954). Determination of Molybdenum in Plant Tissue. Anal. Chem., 26 (3): 572-574.

Kaiser, B. N., K. L. Gridley, J. Ngaire Brady, T. Phillips and S. D. Tyerman (2005). The role of molybdenum in agricultural plant production. Ann. Bot., 96(5):745-754.

Klute, A. (1986). Methods of Soil Analysis, 2nd ed. American Society of Agronomy, Madison, Wisconsin, $183 \mathrm{p}$.

Konstantopoulou, E., G. Kapotis, G. Salachas, S. A. Petropoulos, I. C. Karapanos and H. C. Passam (2010). Nutritional quality of greenhouse lettuce at harvest and after storage in relation to $\mathrm{N}$ application and cultivation season. Sci. Hortic. 125, (93): e1-93.e5.

Lee, S. A., J. H. Fowke, W. Lu, C. Ye, Y. Zheng, K. Gu, Y. T. Gu, X. O. Gao, X. Shu, and W. Zheng (2008). Cruciferous vegetables, the GSTP1 IIe105Val genetic polymorphism, and breast cancer risk. Am. J. clinnutr., 87: 753-760.

Leghari, S. J., N. A. Wahocho, G. M Laghari, A. H. Laghari, G. M. Bhabhan, K. H. Talpur and A. A. Lashari (2016). Role of nitrogen for plant growth and development: A review. Adv. Env. Bio., 10 (9): 209-219.

Lewis, D. H. (1980). Are there inter-relations between the metabolic role of boron, synthesis of phenolic phytoalexins and the germination of pollen? New Phytol., 84: 261-270. 
Liakopoulos, G. and G. Karabourniotis (2005). Boron deficiency and concentrations and composition of phenolic compounds in Olea europaea leaves: a combined growth chamber and field study. Tree physiol., 25 (3): 307-315.

Lichenthaler, H. K. and W. R. Wellburn (1983). Determination of total carotenoids and chlorophylls a and $\mathrm{b}$ of leaf extracts in different solvents. Biochem. Soc. Trans. 11: 591-592.

Lisiewska, Z. and W. Kmiecik (1996). Effects of level of nitrogen fertilizer, processing conditions and period of storage of frozen broccoli and cauliflower on vitamin C retention. Food Chem., 57(2): 267-270.

Markovic, V. and M. Djurovka. (1990). The effect of mineral nutrition on the yield and quality of cauliflower. Acta Hortic. 267: 101-109.

Mazumdar, B. C. and K. Majumder (2003). Methods on Physico-chemical Analysis of Fruits. Daya Publishing House, Delhi-110035, pp.93-95.

Mehrotra, O. N. and P. H. Mishra (1974). Micronutrient deficiencies in cauliflower (Brassica oleracea L. var. botrytis L.). Prog Hortic.

Mitra, S. K., M. K. Sadhu and T. K. Bose (1990). Nutrition of Vegetable crops. Naya Prokash; Calcutta -700006, India. pp. 157-160.

Ningawale, D. K., R. Singh, U.S. Bose, P. S. Gurjar, A. Sharma and U. S. Gautam (2016). Effect of boron and molybdenum on growth, yield and quality of cauliflower (Brassica oleracea var. botrytis) cv. Snowball 16. Indian J. of Agric. Sci., 86(6): 825-829.

Novozamsky, I. and R. V. Eck (1977). Total Sulphur determination in plant material. Z. Anal. Chem. 286: 367-368.

Page, A. L., R. H. Miller and D. R. Keeney (1982). Methods of Soil Analysis. Part 2: Chemical and Microbiological Properties. ASA, Madison, WI.

Picchi, V., C. Migliori, R. L. Scalzo, G. Campanelli, V. Ferrari and L. F. Di Cesare (2012). Phytochemical content in organic and conventionally grown Italian cauliflower. Food Chem., 130(3): 501-509.

Podsedek, A. (2007). Natural antioxidants and antioxidant capacity of Brassica vegetables: A review. LWT Food Sci. Tech., 40(1): 1-11.

Rani, S. N. and K. Mallareddy (2007). Effect of different organic manures and inorganic fertilizers on growth, yield and quality of carrot (Daucus carota L.). Karnataka J. Agric. Sci., 20(3): 686-688.

Russel, E. W. (1973). Soil Conditions and Plant Growth. 10th Ed., The English Language Book Society and Longman. $849 \mathrm{p}$.
Sarker, M. M. H., M. Jahiruddin, A. Z. M. Moslehuddin and M. R. Islam (2018). Micronutrient responsiveness of cauliflower, okra, and rice in a pattern in piedmont soil. J. Plant Nutr., 41(11): 1358-1367.

Shaaban, M. M. (2010). Role of boron in plant nutrition and human health. Am. J. Plant Phys., 5(5): 224-240.

Sharma, S. K. (2002). Effect of boron and molybdenum on seed production of cauliflower. Indian J. Hortic., 59(2): 177-180.

Sharma, C. P. (2006). Plant Micronutrients. Science Publishers, Enfield, NH, USA.

Shelp, B. J. and L. Liu (1992). Nutrient uptake by fieldgrown broccoli and net nutrient mobilization during inflorescence development. Plant and Soil 140: 151155.

Singh, G., S. Sarvanan, K. S. Rajawat, J. S. Rathore and G. Singh (2017). Effect of Different Micronutrients on Plant Growth, Yield and Flower Bud Quality of Broccoli (Brassica oleracea var. Italica). Current Agric. Res. J., 5(1): 108-115.

Tang, L., R.Z. Gary, K. Guru, B.M. Kirsten, Y. Zhang, C.B. Ambrosone and S. E. McCann (2008). Consumption of raw cruciferous vegetables is inversely associated with bladder cancer risk. Cancer epidem. biomar., 17: 938-44.

Tariq, M. and C. J. B. Mott (2007). The significance of boron in plant nutrition and environment-a review. J. Agron., 6(1): 1-10.

Thapa, U., P. H. Prasad and R. Rai (2016). Studies on growth, yield and quality of broccoli (Brassica oleracea L. var italica Plenck) as influenced by boron and molybdenum. J. Plant Nutr., 39(2): 261-267.

Thorwarth, P., E. A. Yousef and K. J. Schmid (2018). Genomic Prediction and Association Mapping of Curd-Related Traits in Gene Bank Accessions of Cauliflower. G3: Genes, Genomes, Genetics, 8(2): 707-718.

Tripathi, D. K. and S. Singh, S. Singh, S. Mishra, D.K. Chauhan and N. K. Dubey (2015). Micronutrients and their diverse role in agricultural crops: advances and future prospective. Acta physiol. Plant., 37(7): 139.

Yoldas, F., S. Ceylan, B. Yagmur and N. Mordogan (2008). Effects of nitrogen fertilizer on yield quality and nutrient content in broccoli. J. Plant Nutr. Soil Sci., 31(7): 1333-1343.

Yousef, E. A., C. Lampei and K. J. Schmid (2015). Evaluation of cauliflower genebank accessions under organic and conventional cultivation in Southern Germany. Euphytica, 201(3): 389-400.

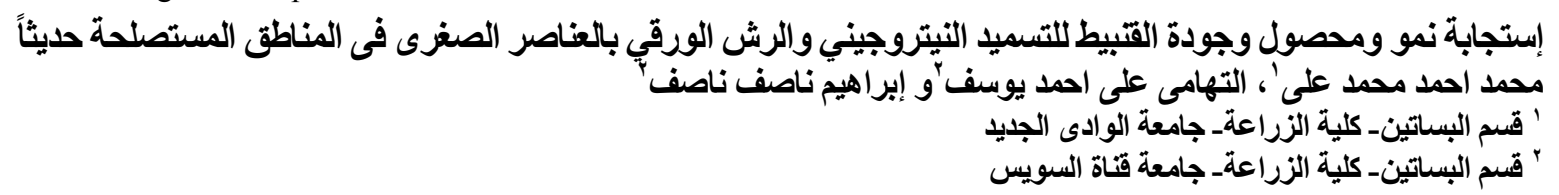

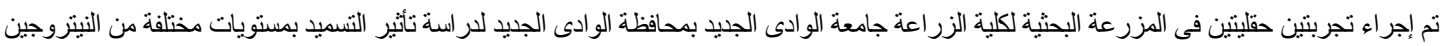

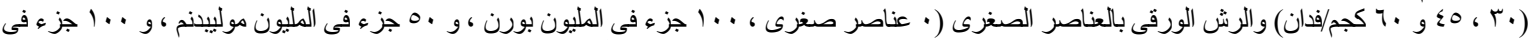

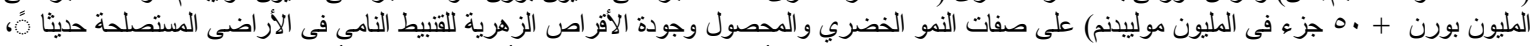

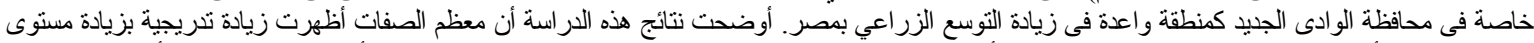

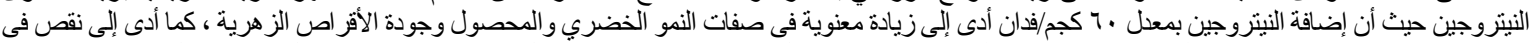

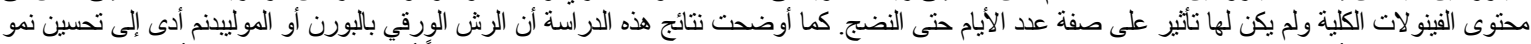

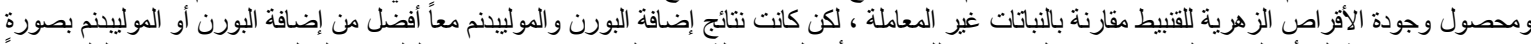

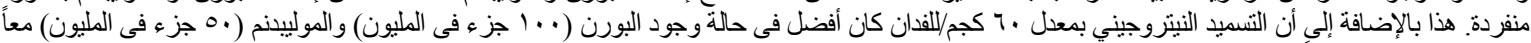

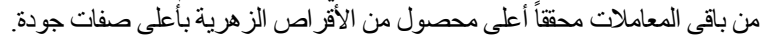

